\title{
Interlayer tunneling and the problem of superfluidity in bilayer quantum Hall systems
}

\author{
D.V. Fil ${ }^{1}$ and S.I. Shevchenko ${ }^{2}$ \\ ${ }^{1}$ Institute for Single Crystal National Academy of Sciences of Ukraine \\ 60 Lenin Ave., Kharkov 61001, Ukraine \\ E-mail: fil@isc.kharkov.ua \\ ${ }^{2}$ B. Verkin Institute for Low Temperature Physics and Engineering of the National Academy of Sciences of Ukraine \\ 47 Lenin Ave., Kharkov 61103, Ukraine \\ E-mail: shevchenko@ilt.kharkov.ua \\ Received January 18, 2007
}

\begin{abstract}
A possibility of nondissipative transmission of electrical current from the source to the load using superfluid electron-hole pairs in bilayers is studied. The problem is considered with reference to quantum Hall bilayers with the total filling factor $v_{T}=1$. At nonzero interlayer tunneling the current pattern looks as a sum of uniform planar counterflow currents and Josephson vortices. The difference of electrochemical potentials of the layers (that is required to support the current in the load circuit) causes the motion of the Josephson vortices. In such a situation the second superfluid viscosity comes into play and results in dissipation of energy. It is found that the power of losses is proportional to the square of the matrix element of the interlayer tunneling and depends nonlinearly on the load resistance.
\end{abstract}

PACS: 73.43.Jn Tunneling;

74.90.+n Other topics in superconductivity.

Keywords: electron-hole superfluidity, Josephson vortices.

Thirty years ago in the papers $[1,2]$ the idea of unusual mechanism of superconductivity that can be realized in electron-hole bilayers was proposed. The idea is based on the fact that in such systems an electron and a hole may form a bound state that can be considered as a composite boson. Superfluid motion of such pairs is equivalent to two nondissipative electrical currents (of opposite direction) in the layers. Using the contacts that allows one to access the layers separately one can realize the counterflow setup and use the bilayer for the transmission of the current from the source to the load. One can expect that such a bilayer will work as a double-wire superconducting transmission line.

The specifics of the electron-hole superfluidity is that the layers should be situated rather close to each other and the interlayer tunneling cannot be neglected. In the presence of tunneling the bilayer system cannot support the uniform planar supercurrent, and the current state becomes a soliton-like [3-5]. This state is similar to the Josephson vortex state in long contacts between two superconductors. The aim of this study is to clarify whether the soliton current state in bilayers remains nondissipative. Our consideration is based on the statement that in the counterflow setup the soliton-like current state becomes nonstationary one. The reason is the following. Nonzero current in the load circuit can appear only if the electrochemical potentials of the layers differ from each other, and in the presence of the electrochemical potential difference the solitons (Josephson vortices) begin to move. We find that such motion results in dissipation of energy.

In this paper the electron-hole superfluidity is studied with reference to a bilayer quantum Hall system. As was pointed out in [6,7] in bilayer systems with the same type of conductivity of the layer subjected by a quantizing magnetic field one can realize the electron-hole superfluidity. The condition is that the sum of filling factors of the layers is equal to unity. The idea [6,7] greatly increases the interest to the experimental and theoretical study of the electron-hole superfluidity. The results of recent experimental investigations of quantum Hall bilayers [8-14] support the theoretical prediction on Bose-Ein- 
stein condensation (BEC) and superfluidity of electron-hole pairs. In particular, in the counterflow experiments a huge increase of longitudinal conductivity was observed $[13,14]$. Other important observations are a strong low bias tunnel conductivity $[8,12]$, a Godstone collective mode [9], and a quantized Hall drag between the layers $[10,11]$. Nevertheless, genuine nondissipative counterflow state was not achieved in the experiments.

The BEC of electron-hole pairs in quantum Hall bilayers can be interpreted as the development of the spontaneous phase coherence between electrons belonging to adjacent layers. The coherent phase $\varphi$ is the phase of the order parameter that describes the electron-hole pairing. The superflow of the pairs is associated with nonzero gradient of the phase. In the continuous approximation the energy of the system can be written as:

$$
E=E_{0}+\int d^{2} r\left[\frac{1}{2} \rho_{s}\left(\frac{d \varphi}{d x}\right)^{2}-\frac{\tilde{t}}{2 \pi \ell^{2}}(\cos \varphi-1)\right],
$$

where we assume that the system is uniform in $y$ direction (direction perpendicular to the current) and neglect the variations of the modulus of the order parameter. In (1) $E_{0}$ is the energy of the ground state, $\tilde{t}=2 t \Delta_{0}$, the tunneling energy ( $t$ is the matrix element of the interlayer tunneling),

$$
\rho_{s}=\Delta_{0}^{2} \frac{e^{2}}{4 \pi \varepsilon \ell}\left[\sqrt{\frac{\pi}{2}} \exp \left(\frac{d^{2}}{2 \ell^{2}}\right) \operatorname{erfc}\left(\frac{d}{\ell \sqrt{2}}\right)\left(1+\frac{d^{2}}{\ell^{2}}\right)-\frac{d}{\ell}\right],
$$

the superfluid stiffness, $\Delta_{0}=\sqrt{v(1-v)}$, the modulus of the order parameter (the filling factors of the layers are $v_{1}=v$ and $\left.v_{2}=1-v\right), d$ is the interlayer distance, $\ell$ is the magnetic length. Eq. (1) is obtained from the equation $E=\langle\Psi|H| \Psi\rangle$, where $H$ is the Hamiltonian of the bilayer system in the lowest Landau level approximation,

$$
|\Psi\rangle=\prod_{X}\left(u_{X}+v_{X} e_{2 X}^{+} h_{1 X}^{+}\right)|v a c\rangle
$$

is the BCS-like many particle wave function, $X$ is the guiding center of the orbit, $e_{i X}^{+}\left(h_{i X}^{+}\right)$, the creation operators for the electron (hole) in the $i$ th layer, the vacuum state $|v a c\rangle$ is defined as the state with empty layer 2 and completely filled $\left(v_{1}=1\right)$ layer 1 .

Varying the energy (1) with respect to the phase and equating the result to zero one obtains the stationary continuity equation

$$
-\frac{e \rho_{s}}{\hbar} \frac{d^{2} \varphi}{d x^{2}}+\frac{e}{\hbar} \frac{\tilde{t}}{2 \pi \ell^{2}} \sin \varphi=0,
$$

where the first term is the divergence of the planar supercurrent density

$$
j_{1}^{(s)}=-j_{2}^{(s)}=-\frac{e \rho_{s}}{\hbar} \frac{d \varphi}{d x},
$$

and the second term is the density of interlayer supercurrent, flowing from the layer 1 to the layer 2 .

$$
I_{1 \rightarrow 2}^{(T)}=\frac{e}{\hbar} \frac{\tilde{t}}{2 \pi \ell^{2}} \sin \varphi
$$

Equation (3) coincides in form with the equation of motion of nonlinear pendulum. At given input current (current from the source) the solution of (3) should satisfy the boundary condition $j_{1}^{(s)}(0)=j_{\text {in }}$, and it should correspond the conditional minimum of energy (1). Note that in difference with the pendulum problem, energy (1) does not coincide with the integral of motion of (3). Using the known solutions for the pendulum problem we find that there is a critical input current $j_{c}=2 e \rho_{s} / \hbar \lambda$ (where $\lambda=\ell \sqrt{2 \pi \rho_{s} \widetilde{t}}$ is the Josephson length) that separates two regimes.

1. At $j_{\text {in }}<j_{c}$ the planar current $j_{1}^{(s)}=$ $=j_{c} \operatorname{sech}\left(\left(x_{0}+x\right) / \lambda\right)\left(x_{0}=\lambda \cosh ^{-1}\left(j_{c} / j_{\text {in }}\right)\right)$ decreases exponentially with distance from the source edge, and for long systems $L_{x} \gg \lambda$ the current at the load edge will be exponentially small. Therefore, at small input current the bilayer system cannot work as the transmission line.

2. At $j_{\text {in }}>j_{c}$ the planar current $j_{1}^{(s)}=\left(j_{c} / \sqrt{\eta}\right) \mathrm{dn} \times$ $\times(x / \lambda \sqrt{\eta}, \eta)\left(\eta=j_{c}^{2} / j_{\text {in }}^{2}, \operatorname{dn}(x, k)\right.$ is the Jacobi elliptic function) contains the constant part and spatially oscillating part. The total current is a sum of the uniform current and the current of Josephson vortices. The planar current reaches the load edge and the bilayer system works as the transmission line.

In the counterflow setup the current withdrawn from one layer should be redirected into the other layer through the load circuit. To support the current in the load circuit the difference of electrochemical potential of the layer is required. At such difference the phase of the order parameter changes in time and the current state becomes nonstationary.

We describe the nonstationary state by the set of two equations for the phase and for the local difference of the electrochemical potentials $V$. The first equation is the nonstationary continuity equation

$$
e \frac{\partial \widetilde{n}}{\partial t}+\frac{\partial j_{1}^{(s)}}{\partial x}+I_{1 \rightarrow 2}=0
$$

where $\tilde{n}$ is the excess local density of the electron-hole pairs that corresponds to the excess local charge densities in the layers. It is connected with $V$ by the capacitor equation $e \tilde{n}=C V$ with

$$
C=\frac{\varepsilon}{4 \pi d}\left\{1-\frac{\ell}{d} \sqrt{\frac{\pi}{2}}\left[1-\exp \left(\frac{d^{2}}{2 \ell^{2}}\right) \operatorname{erfc}\left(\frac{d}{\ell \sqrt{2}}\right)\right]\right\}^{-1},
$$


the effective capacity of the bilayer system per unit area. In Eq. (6) and below we neglect the normal component of the gas of electron-hole pairs.

The second equation is the generalized Josephson equation in which dissipative terms are taken into account. Without derivation this equation was given in [15]. One can justify this equation basing on general arguments. In the superfluid hydrodynamics [16] the equation for the superfluid velocity (in linear approximation) with dissipative terms has the following form:

$$
\frac{\partial \mathbf{v}_{s}}{\partial t}=-\nabla \mu_{m}+\nabla\left[\zeta_{3} \nabla \cdot n_{s} \mathbf{v}_{s}\right]
$$

where $\mu_{m}$ is the chemical potential per unit mass $\mu_{m}=\mu / m$ ( $m$ is the mass of the Bose particle), $n_{s}$ is the superfluid density, $\zeta_{3}$ is the second viscosity for the superfluid component.

To apply Eq. (7) for a description of the bilayer electron-hole superfluidity we should replace the $3 \mathrm{D}$ divergence of the density of the flow with the $2 \mathrm{D}$ divergence of the planar supercurrent plus the term accounted for the leakage caused by the the tunnel supercurrent: $\nabla\left(n_{s} \mathbf{v}_{s}\right) \Rightarrow(-1 / e)\left(\partial j_{1}^{(s)} / \partial x+I_{T, 1 \rightarrow 2}\right)$. The chemical potential should be replaced with the difference of electrochemical potentials $\mu \Rightarrow-e V$. Using Eqs. (4), (5) and the definition of the superfluid velocity $\mathbf{v}_{s}=\hbar \nabla \varphi / m$ ( $m$ is the mass of the electron-hole pairs) we obtain from (7) the following equation

$$
\hbar \frac{\partial \varphi}{\partial t}=e V+\alpha_{\varphi}\left(2 \pi \ell^{2} \rho_{s} \frac{\partial^{2} \varphi}{\partial x^{2}}-\tilde{t} \sin \varphi\right),
$$

where $\alpha_{\varphi}$ is dimensional less parameter proportional to the second viscosity. It is just the equation given in [15]. Equation for the phase that coincides in form with (8) was also derived in [17] for the electron-hole bilayers in zero magnetic field.

Note that nonzero second viscosity does not yield automatically the dissipation. In particular, one can see from Eq. (8) that the viscosity term is equal to zero if the stationary continuity equation (3) is fulfilled. We will show that under motion of Josephson vortices the second viscosity results in dissipation.

We consider the case of small dissipation when the power of losses is much smaller than the input power. We restrict our consideration to the case of large input current (much larger than $j_{c}$ ). At large input current the approximate solution of Eqs. (6), (8) can be presented in the form

$$
\varphi(x, t)=\omega t-k x+A_{\varphi} \sin (\omega t-k x)+B_{\varphi} \cos (\omega t-k x),
$$

$$
V(x, t)=V_{0}\left[1+A_{V} \sin (\omega t-k x)+B_{V} \cos (\omega t-k x)\right]
$$

where $V_{0}$ is the average difference of the electrochemical potentials of the layers, $\omega=e V_{0} / \hbar$, and $k$ is wave number connected with the average planar supercurrent by the relation $j_{0}=e \rho_{s} k / \hbar$. The presence of oscillating terms in (9), (10) is caused by the interlayer tunneling: all the coefficients $A_{\varphi}, B_{\varphi}, A_{V}, B_{V}$ are proportional to $\widetilde{t}$. The explicit form of these coefficient can be obtained by substituting of (9), (10) into (6), (8) and taking into account the term linear in $\tilde{t}$. The solution (9), (10) corresponds the vortex structure that moves with the velocity $v_{v}=\omega / k=$ $=e^{2} \rho_{s} R_{l} L_{y} / \hbar^{2}\left(V_{0}\right.$ and $j_{\text {out }} \approx j_{0}$ satisfy the Ohm's law $V_{0}=j_{\text {out }} L_{y} R_{l}$, where $R_{l}$ is the load resistance).

The power of losses for the transmission line is determined as the difference between the input and the output power $P_{l}=\left(j_{\text {in }}-j_{\text {out }}\right) L_{y} V_{0}$. The difference $j_{\text {in }}-j_{\text {out }}$ emerges if the average interlayer current $\bar{I}_{T}$ becomes nonzero: $j_{\text {in }}-j_{\text {out }}=\bar{I}_{T} L_{x}$. Thus, the power of losses per unit area is proportional to the average interlayer current: $p_{l}=P_{l} / L_{x} L_{y}=\bar{I}_{T} L_{x}$ (for the case of small dissipation). One can see from Eq. (4) that the average interlayer current is proportional to $B_{\varphi}: \bar{I}_{T}=e \widetilde{t} B_{\varphi} / 4 \pi \ell^{2} \hbar$. The explicit expression for $B_{\varphi}$ reads as

$$
B_{\varphi}=\frac{\tilde{t}}{2 \pi \ell^{2} k^{2} \rho_{s}} \alpha_{\varphi} \frac{V_{0}}{V_{C}} \frac{\beta^{2}}{\left(1-\beta^{2}\right)^{2}+\alpha_{\varphi}^{2} V_{0}^{2} / V_{C}^{2}},
$$

where $\beta^{2}=C V_{0}^{2} / \rho_{s} k^{2}$ and $V_{C}=e / 2 \pi \ell^{2} C$. As follows from (11) $B_{\varphi} \neq 0$ and $p_{l} \neq 0$ due to nonzero second viscosity $\alpha_{\varphi} \propto \zeta_{3}$. We emphasize that our analysis is not valid for $\beta$ that corresponds $R_{l} \rightarrow R_{c}=\hbar /\left(e L_{y} \sqrt{\rho_{s} C}\right)$. At such $R_{l}$ the dissipation is large. At $R_{l}$ not very close to $R_{c}$ the power of losses is given by the equation

$$
p_{l}=\alpha_{\varphi} \frac{\tilde{t}^{2}}{4 \pi \ell^{2} \hbar} \frac{\left(\frac{R_{l}}{R_{c}}\right)^{4}}{\left(1-\left(\frac{R_{l}}{R_{c}}\right)^{2}\right)^{2}}
$$

Thus, the power of losses is proportional to the square of the tunneling amplitude and depends nonlinearly on the load resistance.

In conclusion, we have shown that in bilayer systems with interlayer phase coherence the dissipation may appear under the transmission of the current from the source to the load in the counterflow regime. The dissipation is caused by joint action of two factors. The first factor is nonzero interlayer tunneling. The tunneling results in a formation of the vortex current state. The second factor is the motion of vortices. They begin to move due to the difference of electrochemical potentials between the layers. The dissipation emerges due to second viscosity that comes into play only for moving vortices. Since one of the factors is the motion of vortices, one can hope that the dissipation can be eliminated by vortex pinning. 
1. Yu.E. Lozovik and V.I. Yudson, Zh. Eksp. Theor. Fiz. 71, 738 (1976) [Sov. Phys. JETP 44,389 (1976)].

2. S.I. Shevchenko, Fiz. Nizk. Temp. 2, 505 (1976) [Sov. J. Low Temp. Phys. 2 , 251 (1976)].

3. S.I. Shevchenko, Fiz. Nizk. Temp. 3, 605 (1977) [Sov. J. Low Temp. Phys. 3, 293 (1977)].

4. S.I. Shevchenko, Phys. Rev. Lett. 72, 3242 (1994).

5. M. Abolfath, A.H. MacDonald, and L. Radzihovsky, Phys. Rev. B68, 155318 (2003).

6. H. MacDonald and E.H. Rezayi, Phys. Rev. B42, 3224 (1990).

7. X.G. Wen and A. Zee, Phys. Rev. Lett. 69, 1811 (1992).

8. I.B. Spielman, J.P. Eisenstein, L.N. Pfeiffer, and K.W. West, Phys. Rev. Lett. 84, 5808 (2000).

9. I.B. Spielman, J.P. Eisenstein, L.N. Pfeiffer, and K.W. West, Phys. Rev. Lett. 87, 036803 (2001).

10. M. Kellogg, I.B. Spielman, J.P. Eisenstein, L.N. Pfeiffer, and K.W. West, Phys. Rev. Lett. 88, 126804 (2002).
11. M. Kellogg, J.P. Eisenstein, L.N. Pfeiffer, and K.W. West, Phys. Rev. Lett. 90, 246801 (2003).

12. I.B. Spielman, M. Kellogg, J.P. Eisenstein, L.N. Pfeiffer, and K.W. West, Phys. Rev. B70, 081303 (2004).

13. M. Kellogg, J.P. Eisenstein, L.N. Pfeiffer, and K.W. West, Phys. Rev. Lett. 93, 036801 (2004)

14. E. Tutuc, M. Shayegan, and D.A. Huse, Phys. Rev. Lett. 93, 036802 (2004).

15. A.H. MacDonald, A.A. Burkov, Y.N. Joglekar, and E. Rossi, Physica E22, 19 (2004).

16. S.J. Putterman, Superfluid Hydrodynamics, North-Holland Publ. Comp.,Amsterdam, London, American Elsevier Publ. Comp. Inc. - NY (1974).

17. A.I. Bezuglyj and S.I. Shevchenko, Fiz. Nizk. Temp. 30 282 (2004) [Low Temp. Phys. 30, 209 (2004)]. 